\title{
Midterm results of Manouguian double valve replacement: Comparison with standard double valve replacement
}

Hiroshi Okuyama, MD

Kazuhiro Hashimoto, MD

Hiromi Kurosawa, $\mathrm{MD}^{\mathrm{b}}$

Kei Tanaka, MDa

Yoshimasa Sakamoto, MD

Kazuaki Shiratori, MD ${ }^{\mathrm{a}}$

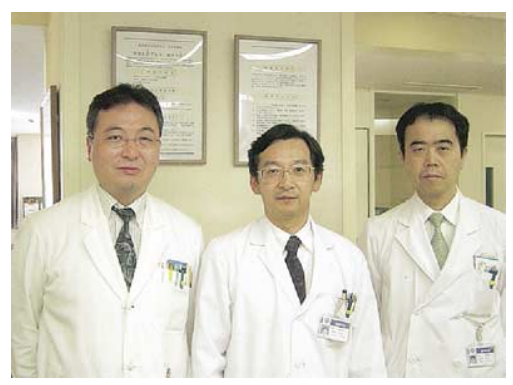

Okuyama, Hashimoto, Sakamoto (left to right)
Objective: Surgical strategies for patients who have a small aortic annulus associated with a small mitral annulus remain controversial. The objective of this study was to assess the validity of the Manouguian procedure for double valve replacement with patch enlargement of the annuli.

Methods: We reviewed 57 consecutive patients who underwent double valve replacement for combined aortic and mitral disease between September 1991 and October 2000. Thirty patients underwent the Manouguian procedure with patch enlargement of the aortic and mitral annuli, and the other 27 patients had standard double valve replacement. The Manouguian procedure was selected for a small aortic annulus of less than $21 \mathrm{~mm}$ in diameter. The patients were followed for a median of 7.5 years. Results of echocardiography and the dobutamine stress test were compared postoperatively.

Results: Double valve replacement with the Manouguian procedure did not increase the early or midterm mortality because the survival (including operative death) at 8 years was $83 \%$ in the Manouguian group and $84 \%$ in the double valve replacement group $(P=.82)$. The event-free rate at 8 years was $79 \%$ in the Manouguian group and $84 \%$ in the double valve replacement group $(P=.6)$. The prostheses implanted at the aortic and mitral positions were smaller in the Manouguian group, even after annular enlargement. However, the transprosthetic gradient across the aortic valve evaluated by means of dobutamine stress echocardiography did not differ between the 2 groups, possibly because of a smaller body surface area in the Manouguian group.

Conclusions: The Manouguian patch enlargement procedure is useful during double valve replacement when associated with the problem of small-valve annuli.

\footnotetext{
From the Department of Cardiovascular Surgery, ${ }^{a}$ Jikei University School of Medicine, and the Heart Institute, ${ }^{\mathrm{b}}$ Tokyo Women's Medical University, Tokyo, Japan.

Received for publication Sept 23, 2004; revisions received Oct 18, 2004; accepted for publication Oct 28, 2004.

Address for reprints: Kazuhiro Hashimoto, MD, Department of Cardiovascular Surgery, Jikei University School of Medicine, 3-25-8, Nishishinbashi, Minatoku, Tokyo, Japan 105-8461 (E-mail: kaz-hashi@jikei. ac.jp).

J Thorac Cardiovasc Surg 2005;129:869-74 0022-5223/\$30.00

Copyright (C) 2005 by The American Association for Thoracic Surgery

doi:10.1016/j.jtcvs.2004.10.026
}

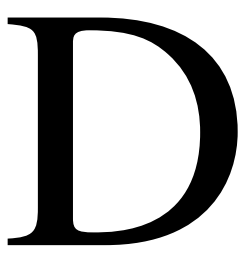

ouble valve replacement (DVR), replacement of both the aortic and mitral valves, has been accomplished easily and safely in recent years. However, combined valvular disease is usually of rheumatic origin in Japan and is often associated with the problem of small-valve annuli, particularly the aortic annulus. When mitral valve disease is also associated with a small mitral annulus, patients might experience prosthesis mismatch after ordinary DVR, leading to persistent or progressive left ventricular dysfunction. At our hospital, we have performed enlargement of both the aortic and mitral annuli with a patch to accept a larger valve prosthesis using the Manouguian (DVR) procedure. ${ }^{1}$ In recent years, high-performance prosthetic valves have been introduced, ${ }^{2-4}$ and this development might eliminate the need for aggressive enlargement of the valve orifice. However, 
TABLE 1. Preoperative (original) disease

\begin{tabular}{|c|c|c|c|c|c|c|c|c|}
\hline & \multicolumn{4}{|c|}{ Aortic valve } & \multicolumn{4}{|c|}{ Mitral valve } \\
\hline & AS & ASR & AR & IE & MS & MSR & MR & IE \\
\hline Manouguian $(\mathrm{n}=30)$ & 15 & 8 & 7 & $A R(1)$ & 17 & 6 & 7 & MR (1) \\
\hline \multirow[t]{2}{*}{ Standard DVR $(\mathrm{n}=27)$} & 2 & 11 & 14 & $A R(1)$ & 7 & 10 & 9 & 1 \\
\hline & \multicolumn{4}{|c|}{ Manouguian vs DVR: $P<.01$} & \multicolumn{4}{|c|}{ Manouguian vs DVR: $P=.08$} \\
\hline
\end{tabular}

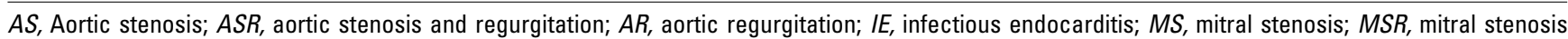
and regurgitation; $M R$, mitral regurgitation; $D V R$, double valve replacement.

\section{TABLE 2. Preoperative patient characteristics}

\begin{tabular}{lccc}
\hline & Manouguian (30) & Standard DVR (27) & P value \\
\hline Male/female & $10 / 20$ & 17,10 & .05 \\
Age (median/min-max), y & $56 / 37-72$ & $55 / 33-72$ & $\mathrm{NS}$ \\
BSA (median/min-max), cm & $1.48 / 1.14-1.88$ & $1.54 / 1.07-2.06$ & $\mathrm{NS}$ \\
Previous operations (\%) & 57 & 22 & .05 \\
PTMC (n) & 1 & 1 & \\
CMC (n) & 3 & 3 & \\
OMC (n) & 10 & 1 & \\
AVR (n) & 1 & 0 & \\
MVR (n) & 2 & 1 & \\
DVR (n) & 0 & 16 & NS
\end{tabular}

DVR, Double valve replacement; min-max, minimum-maximum; $N S$, not significant; $B S A$, body surface area; PTMC, percutaneous transluminal mitral commissurotomy; $C M C$, closed mitral commissurotomy; $O M C$, open mitral commissurotomy; $A V R$, aortic valve replacement; $M V R$, mitral valve replacement.

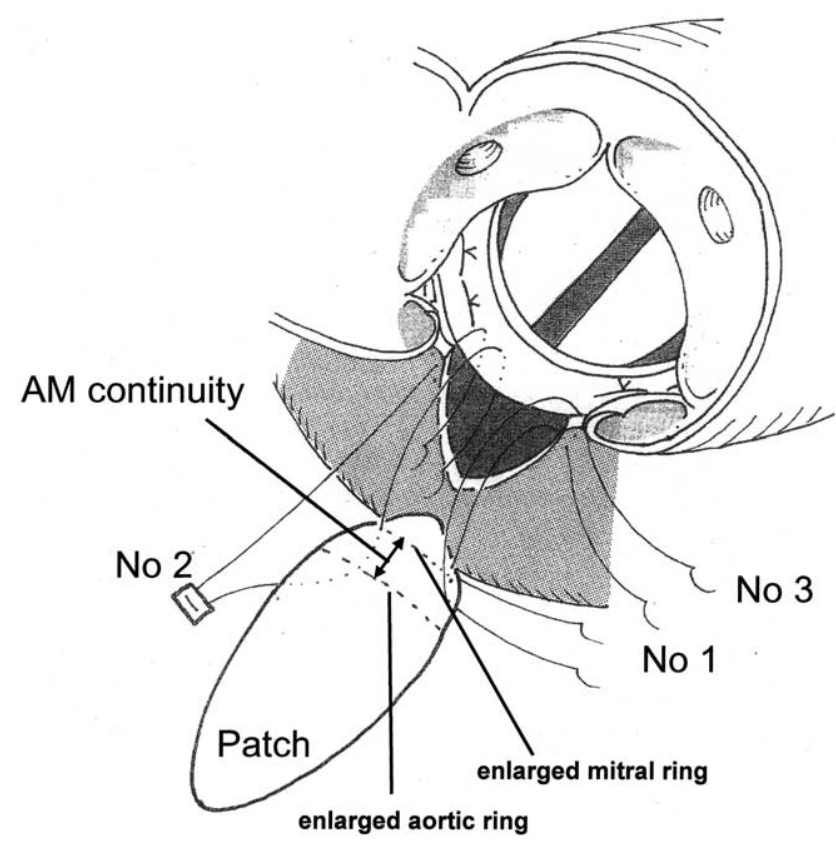

Figure 1. Manouguian annular enlargement.

some patients might still require the Manouguian procedure, despite newer prostheses. Since the Manouguian technique (DVR) was introduced, only a few case reports about it have been published, ${ }^{5-8}$ and no follow-up data are available, except one report on a small number of patients. ${ }^{9}$
In this study we investigated the early and midterm results of Manouguian DVR and made a retrospective comparison with standard DVR. The pressure gradient across the aortic valve prosthesis was also assessed by means of echocardiography. On the basis of our findings, we assessed the validity of this technique.

\section{Patients and Methods}

Patient records and the clinical database were reviewed retrospectively for all 57 consecutive patients who underwent replacement of the aortic and mitral valves between September 1991 and October 2000. Thirty patients underwent DVR with Manouguian annulus enlargement (Manouguian group), and the other 27 patients underwent standard DVR (DVR group). A St Jude Medical mechanical prosthesis (Standard; St Jude Medical, Inc, St Paul, Minn) was used in the aortic position, and a Carbomedics mechanical prosthesis (Sulzer Carbomedics, Inc, Austin, Tex) was used in the mitral position for all patients because this was our policy at that time. All valves were placed in the intra-annular position. The Manouguian procedure was selected for a small aortic valve orifice of less than $21 \mathrm{~mm}$ in diameter on direct measurement with valve sizers, except in patients with infective endocarditis. The cause of valve disease was rheumatic in all but 2 patients with infective endocarditis, and the details are listed in Tables 1 and 2 .

The midterm survival and freedom from events (death [including operative death], reoperation, cardiac events, and thromboembolic episodes) were reviewed in both groups. Transthoracic echocardiography was carried out at 1 to 3 months after surgical intervention in all patients, and the prosthetic valve pressure gra- 
TABLE 3. Intraoperative data

\begin{tabular}{|c|c|c|c|}
\hline & Manouguian (30) & Standard DVR (27) & $P$ value \\
\hline Median aortic diameter (median/min-max), mm & 20/17-23 & 24/23-25 & .05 \\
\hline GOAI of AVP (median/min-max), $\mathrm{cm}^{2} / \mathrm{m}^{2}$ & $1.79 / 1.58-2.28$ & $1.82 / 1.5-2.43$ & NS \\
\hline Ao clamp time (median/min-max), min & $189 / 136-290$ & 122/89-177 & .05 \\
\hline
\end{tabular}

$D V R$, Double valve replacement; min-max, minimum-maximum; GOAl, geometric orifice area index; $A V P$, aortic valve prosthesis; NS, not significant; $A 0$, aortic.

TABLE 4. Size of prostheses used in aortic and mitral position

\begin{tabular}{|c|c|c|c|c|c|c|c|}
\hline & \multicolumn{3}{|c|}{ Aortic position } & \multicolumn{4}{|c|}{ Mitral position } \\
\hline & $21 \mathrm{~mm}$ & $23 \mathrm{~mm}$ & $25 \mathrm{~mm}$ & $27 \mathrm{~mm}$ & $29 \mathrm{~mm}$ & $31 \mathrm{~mm}$ & $33 \mathrm{~mm}$ \\
\hline Manouguian $(\mathrm{n}=30)$ & 1 & 26 & 3 & 2 & 19 & 9 & 0 \\
\hline \multirow[t]{2}{*}{ Standard DVR $(\mathrm{n}=27)$} & 4 & 12 & 11 & 4 & 8 & 14 & 1 \\
\hline & \multicolumn{3}{|c|}{ Manouguian vs DVR: $P<.01$} & \multicolumn{4}{|c|}{ Manouguian vs DVR: $P=.06$} \\
\hline
\end{tabular}

DVR, Double valve replacement.

dient was also evaluated by means of dobutamine stress echocardiography in 20 volunteers (not intentionally selected) from each group. Doppler echocardiography was performed at our institute by the same group of echocardiographers who were unaware of the operations performed on the patients. Flow velocity in the left ventricular outflow tract and across the valve was measured by means of pulsed and continuous wave Doppler ultrasonography, respectively. Then the modified Bernoulli equation was used to calculate the mean pressure gradient across the prosthesis. For dobutamine stress echocardiography, baseline data were obtained first. Then dobutamine was infused through a peripheral vein at incremental doses of 3,6 , and $9 \mu \mathrm{g} \cdot \mathrm{kg}^{-1} \cdot \mathrm{min}^{-1}$ at 10 -minute intervals, and the transaortic prosthetic mean pressure gradient was measured with each dose of dobutamine. Doppler measurements were obtained and averaged over 5 representative beats.

\section{Operative Procedure and Anticoagulation}

General anesthesia was induced and maintained with fentanyl citrate and an inhalant (isoflurane or enflurane). Muscle relaxation was achieved with pancuronium bromide. Cardiopulmonary bypass was established by means of direct cannulation of the vena cava and the aorta. Body temperature was allowed to drift spontaneously without active cooling. Cold blood cardioplegia was used in both the antegrade and retrograde directions according to the integrated myocardial protection method introduced by Buckberg. ${ }^{10}$ A terminal hot shot was also used. DVR was performed in the standard fashion, and the Manouguian procedure was based on the original method, ${ }^{1}$ with some technical amendments according to our previous report. ${ }^{11}$ Briefly, about three quarters of the circumference of the mitral prosthesis was fixed to the native mitral ring with 2-0 polyester mattress sutures. Next an equine pericardial patch shaped like the hull of a ship and lined with a Dacron fabric patch (about $3 \times 6 \mathrm{~cm}$ ) was stitched to the remainder of the mitral valve ring and the prosthesis. The part used for aortic annulus enlargement $(40 \mathrm{~mm})$ was larger than that used for the mitral ring (25 $\mathrm{mm}$ ), and a distance of $1 \mathrm{~cm}$ was kept between them, which is equivalent to the size of the aortic-mitral (AM) continuity. Stitch

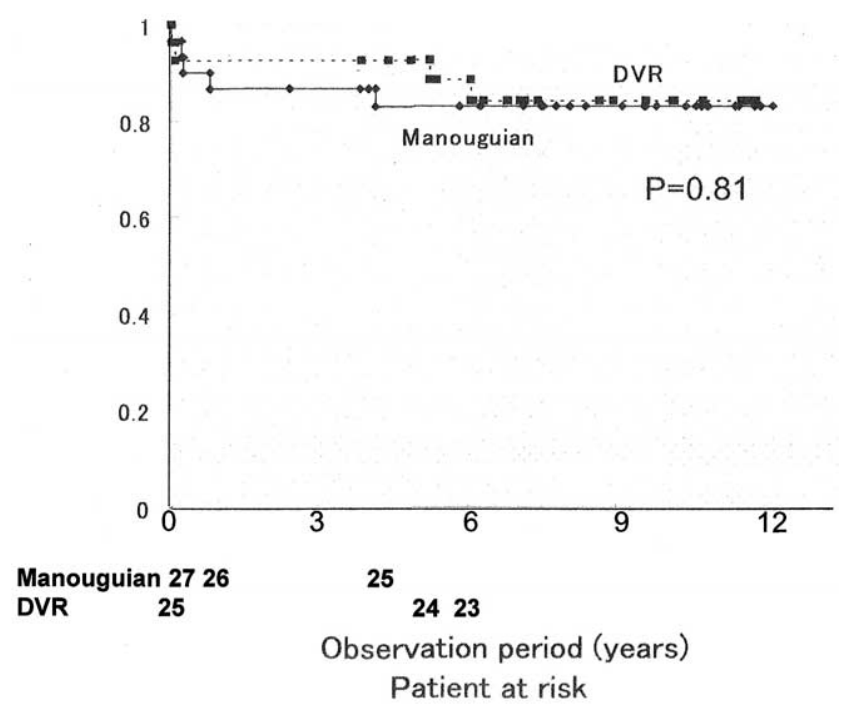

Figure 2. Survival curve of the Manouguian group and standard DVR group.

no. 1 was placed for closure of the left atrium. Stitch no. 2 was a transitional stitch for approximating the left atrium, the AM continuity, and the prosthetic valve. Stitch no. 3 was used to reconstruct the AM continuity and the aorta with the patch. By means of suturing like this, the patch could be fitted precisely (Figure 1). Patch closure of the left atrial superior wall was then done with a running 4-0 polypropylene suture. Next one quarter of the residual mitral valve cuff was secured to the patch with 2-0 polyester mattress sutures. A running suture was inserted along the aortic side of the patch with 4-0 polypropylene sutures from the AM continuity to the aortic wall. The aortic valve prosthesis was fixed to the aortic annulus, and the aortic part of the patch was fixed with 2-0 polyester mattress sutures. After trimming the patch, the aortic wall was finally closed with continuous double running 4-0 polypropylene sutures. 


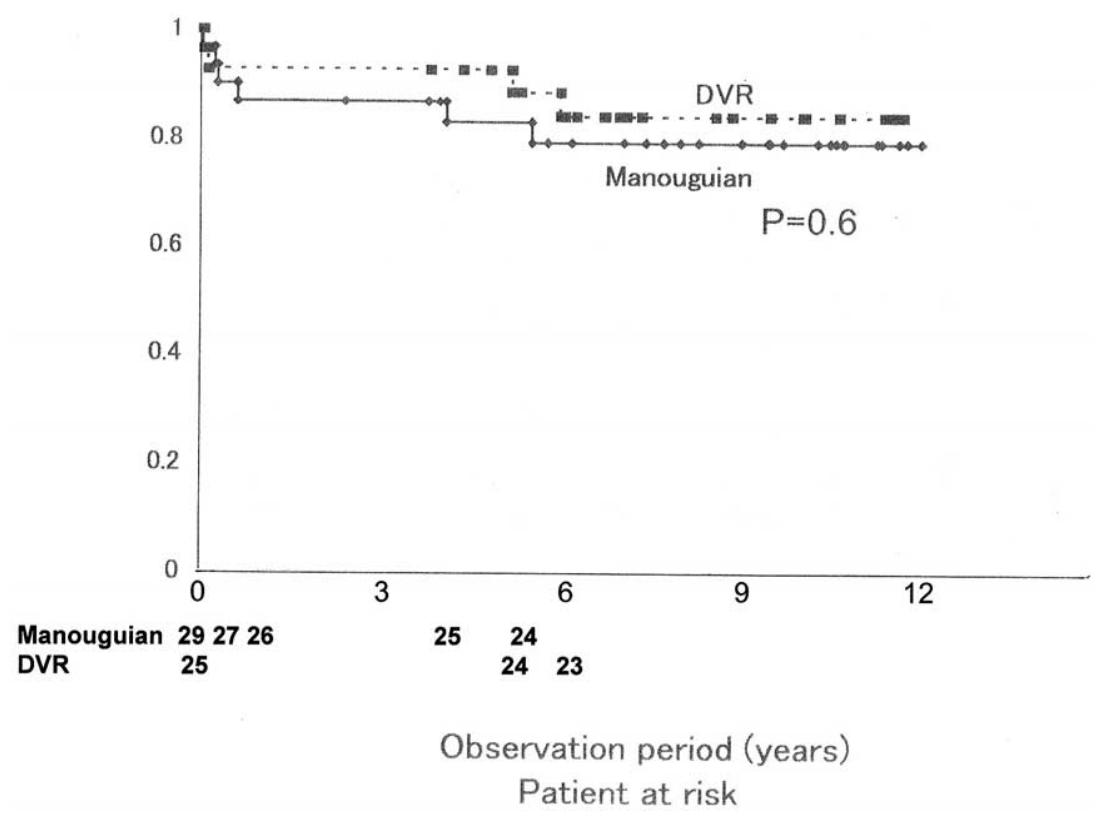

Figure 3. Freedom from events in the Manouguian group and standard DVR group.

All patients were given warfarin and an antiplatelet agent (100 $\mathrm{mg} / \mathrm{d}$ aspirin or $200 \mathrm{mg} / \mathrm{d}$ tichropidine) postoperatively. The prothrombin time was maintained at a target international normalized ratio of 2.1 to 2.5 .

\section{Statistical Analysis}

Statistical analysis was done with the SAS program (SAS Institute, Inc, Cary, NC) on a Windows computer. Values are given as the median and range. Patient characteristics and hospital outcomes were compared by using the unpaired $t$ test for continuous variables and the $\chi^{2}$ test or the Fisher exact test for categorical variables. Continuous variables at the dobutamine stress test were analyzed by repeatedmeasures analysis of variance with the Fisher post-hoc test. Late survival and freedom from events were evaluated by the KaplanMeier method. Statistical significance of differences in the KaplanMeier curves was determined by the log-rank test.

\section{Results}

Stenotic lesions in the aortic position were much more common in the Manouguian group (Table 1), and the number of patients with previous operations or female patients was also significantly greater in the Manouguian group (Table 2). The age and presence of atrial fibrillation did not differ between the groups. However, the body surface area of the Manouguian group was somewhat smaller, although the difference was not significant (Table 2). The median follow-up period was 7.5 years, ranging from 1 day to 4423 days.

The intraoperative aortic annular diameter of the Manouguian group was significantly smaller than that of the DVR group, but the geometric orifice area divided by the body surface area of the aortic valve was not different. The aortic crossclamp time of the Manouguian group was about 60 minutes longer than that of the standard DVR group
(Table 3). The prostheses implanted at the aortic and mitral positions were smaller in the Manouguian group (Table 4), but only 1 patient undergoing the Manouguian procedure received a 21-mm aortic valve. Operative death (death within 30 days or hospital death after the operation) occurred in $2(6.7 \%)$ patients from the Manouguian group and $2(7.4 \%)$ patients from the standard DVR group. There was no statistical difference in the early mortality rate. Severe early complications, such as the need for reopening of the chest to control bleeding, perivalvular leakage, or hemolysis, were not observed in either group.

The 8-year survival (including operative deaths) was $83 \%$ in the Manouguian group and $84 \%$ in the standard DVR group (Figure 2). Three patients from the Manouguian group died during midterm follow-up because of the recurrence of infectious endocarditis $(n=1)$ or thromboembolism $(n=2)$. Two patients from the standard DVR group died of sudden death $(n=1)$ or thromboembolism $(n=1)$. The event-free rate was $79 \%$ in the Manouguian group and $84 \%$ in the standard DVR group after a median follow-up period of 8 years $(P=.6$, Figure 3$)$. The international normalized ratio was optimal in the patients at that time. In the follow-up period, reoperation was done in 1 patient in the Manouguian group but no patients in the standard DVR group. The one patient who required reoperation in the Manouguian group underwent a modified Bentall procedure for a mycotic aneurysm of the aortic annulus. He finally died 6 months after a third operation. There was no significant difference between the 2 groups with respect to survival, event-free survival, and reoperation. 


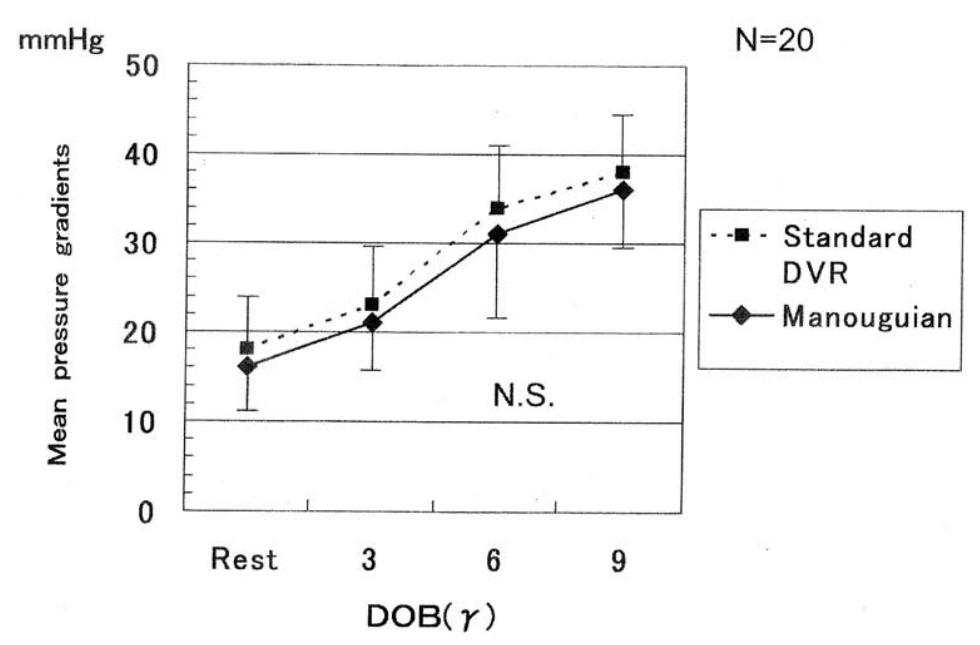

Figure 4. Transprosthetic pressure gradients examined with dobutamine stress echocardiography. There was no significant difference in the transprosthetic mean pressure gradient at the aortic valve between the 2 groups.

Postoperative dobutamine stress echocardiography showed no significant difference of the transprosthetic mean pressure gradient at the aortic valve between the 2 groups (Figure 4 ).

\section{Discussion}

Surgical management of patients with a small aortic annulus remains controversial. Several studies have demonstrated that placing a standard prosthetic aortic valve in a small aortic annulus without performing enlargement leads to at least mild stenosis. ${ }^{12-16}$ High-performance prosthetic valves have been introduced recently to overcome this problem. ${ }^{2-4}$ However, a question still remains about whether such advanced prosthetic aortic valves can avoid significant obstruction. The difficulty of placing a valve in a small aortic annulus becomes much more acute when there is associated mitral valve disease, particularly mitral stenosis. Insertion of a properly matched prosthetic valve at the mitral position first tends to complicate subsequent insertion of the aortic prosthesis in the narrowed aortic annulus. Thus, a small mitral valve prosthesis is usually selected to minimize the difficulty of aortic valve insertion. Patch enlargement of both the aortic and mitral annuli during DVR is an attractive and useful procedure to solve this problem. In 1979, Manouguian ${ }^{17}$ originally reported a technique for aortic valve replacement with enlargement of a small aortic annulus by extending the aortic incision to the anterior mitral leaflet. Patch enlargement of the aortic and mitral annuli with DVR was experimentally introduced at the same time. ${ }^{1}$ The aortic annulus can be enlarged almost as much as needed for the patient (usually an aortic prosthesis that is 2 sizes larger) by this technique, and a properly matched mitral valve prosthesis can be inserted at the same time. Although a few reports on DVR with this method have been published, most of these are only case reports, ${ }^{5-8}$ and there is only one containing data on the early and midterm outcome. ${ }^{9}$ The purpose of the present study was to clarify any technical problems and to assess the late outcome in patients treated at a single institution. We also compared the results with the outcome of standard DVR (aortic and mitral valves) performed during the same period.

Initially, we were concerned that the Manouguian procedure might cause some serious postoperative problems (valve movement, bleeding, hemolysis, leakage, infective endocarditis, or cardiac failure). However, our experience demonstrated a similar operative mortality and morbidity as standard DVR, although the mean aortic crossclamp time was about 1 hour longer in the Manouguian group. Because we noted sticking of the valves during surgical intervention when both valves were placed in a perpendicular direction, the aortic and mitral prostheses were always oriented in parallel to prevent interference with each other. According to Manouguian's experiment, if the AM continuity is enlarged by more than $30 \mathrm{~mm}$ (a 30-mm increase of the mitral ring), the ring of mitral prosthesis protrudes into the left ventricular outflow tract and obstructs it. Thus, after making Manouguian's incision and resection of the native valves, it is important to determine the patch size that is appropriate for enlargement by inserting a valve sizer. In our series the expansion of the mitral annulus was usually around 15 to 20 $\mathrm{mm}$. Also, it is important to keep some distance between the mitral and aortic valve suture lines on the patch.

In addition, we took great care with suturing and approximation at the transitional areas, such as the mitral ring, the mitral prosthetic valve cuff, the patch, the left atrial wall, and the AM continuity. This is a major concern during reconstruction because these are potential sites of leakage and hemolysis. Ensuring an appropriate width compatible with the AM continuity is also important to prevent such problems. By paying 
attention to these points, we could completely avoid hemolysis and perivalvular leakage, which were encountered in the series of Kawachi and associates. ${ }^{9}$

In Kawachi and associate's experience, ${ }^{9} 5$ of 8 patients needed reoperation. Three cases were caused by structural failure of Hancock bioprostheses, but prosthetic valve endocarditis and leakage occurred in one case each. They recommended that bioprostheses should not be used and that mechanical valves were a better choice. This technique could also be applied to reconstruction after debridement of infection or abscesses extending to the intervalvular fibrous trigone in patients with severe acute infective endocarditis. However, this procedure is associated with a significant risk of recurrence of endocarditis, and it should be used in those cases as carefully as possible.

The midterm survival of our patients who had patch enlargement of the aortic and mitral annuli was similar to that of our patients who received standard DVR. The midterm morbidity (reoperation, thromboembolic episodes, prosthetic endocarditis, and other cardiac events) of the Manouguian group was also similar to that of the standard DVR group. Although this series was not a comparative study in patients with small aortic annuli because whether we used Manouguian DVR depended on the size of the aortic annulus, echocardiographic data (pressure gradients across the aortic valve) were similar to those in the DVR group, suggesting that we could avoid patient-prosthesis mismatch. Although the aortic prostheses implanted in the Manouguian group were smaller than those implanted in the standard DVR group, the body surface area was also smaller. As a result, dobutamine stress echocardiography confirmed the appropriateness of our method by demonstrating the same increase of the pressure gradient in the 2 groups.

We conclude that DVR with the Manouguian procedure does not lead to increased early or midterm mortality. Morbidity was also comparable with that after standard DVR. Therefore, we think that the Manouguian procedure is an appropriate option to improve patient-prosthesis mismatch, not only for the aortic valve but also for the mitral valve.

\section{References}

1. Manouguian S, Abu-Aishah N, Neuzel J. Patch enlargement of the aortic and mitral valve rings with aortic and mitral double valve replacement. J Thorac Cardiovasc Surg. 1979;78:394-401.
2. Zingg U, Aeschbacher B, Seiler C, Althaus U, Carrel T. Early experience with the new Master series of St. Jude medical heart valve: in vivo hemodynamic and clinical results in patients with narrowed aortic annulus. J Heart Valve Dis. 1997;6:535-41.

3. Bach DS, Goldbach M, Sakwa MP, Petracek M, Errett L, Mohr F. SJM Regent Study Group. Hemodynamics and early performance of the St. Jude Medical Regent aortic valve prosthesis. J Heart Valve Dis. 2001;10:436-42.

4. Jazayeri S, Gomez MC, Tatou E, Ecarnot A, Saleh M, Bouchot O, et al. Clinical experience and Doppler echocardiographic assessment of the first one hundred ATS AP (advanced performance) prosthetic valve in the aortic position. J Heart Valve Dis. 2003;12:628-34.

5. Fruse A, Mizuno A, Asano A. Aortoatrioplasty with double valve replacement for infective endocarditis. J Cardiovasc Surg (Torino). 1984;25:462-4.

6. Minatoya K, Okabayashi H, Shimada I, Ohno N, Nishina T, Kameyama T. Mitral and aortic annular enlargement for small mitral annulus after mitral annuloplasty. Nippon Kyoubu Geka Gakkai Zasshi. 1995;43:1077-80.

7. Thongcharoen P, Subtawaeesin T, Sakiyalak P. Double annulus enlargement, Rastan-Manouguian's technique: case report. J Med Assoc Thai. 1998;12:1034-9.

8. Takakura H, Kurosawa H, Onishi K, Okubo H. A case of Manouguian's procedure for aortic stenosis with severe calcification extending on to anterior mitral leaflet. Ann Thorac Cardiovasc Surg. 1995; 2:115-7.

9. Kawachi Y, Tominaga R, Tokunaga K. Eleven-year follow-up study of aortic or aortic-mitral annulus-enlarging procedure by Manouguian's technique. J Thorac Cardiovasc Surg. 1992;104:1259-63.

10. Buckberg GD. Update on current techniques of myocardial protection. Ann Thorac Surg. 1995;60:805-14.

11. Mashiko K, Shimizu S, Okuyama H, Mizuno A, Suzuki K, Nakano M, et al. A technique of successfully performing an annuloplasty for narrowed aortic valvular annulus in aortic valve replacement. $J J p n$ Assoc Thorac Surg. 1994;42:1267-71.

12. Hashimoto K, Mashiko K, Nakano M, Kurosawa H, Arai T. Use of 21 $\mathrm{mm}$ monostrut Bjork-Shiley valve in patients with a narrow aortic root. Cardiovasc Surg. 1994;2:456-9.

13. Sawant D, Singh AK, Feng WC, Bert AA, Rotenberg F. St. Jude medical cardiac valves in small aortic roots: follow up to sixteen years. J Thorac Cardiovasc Surg. 1997;113:499-509.

14. Aoyagi S, Arinaga K, Fukunaga S, Kawano H, Kawara T. Aortic valve replacement with a small mechanical valve prosthesis-a clinical and echocardial study of a St Jude medical valve prosthesis. Jpn Circ J. 1998;62:244-248

15. Niinami H, Aomi S, Tomioka H, Nakano K, Koyanagi H. A comparison of the in vivo performance of the $19-\mathrm{mm}$ St. Jude medical hemodynamic plus and 21-mm standard valve. Ann Thorac Surg. 2002;74:1120-4

16. Milano A, De Carlo M, Mecozzi G, D'Alfonso A, Scioti G. Clinical outcome in patients with $19-\mathrm{mm}$ and $21-\mathrm{mm}$ St Jude aortic prosthesis: Comparison at long term follow-up. Ann Thorac Surg. 2002;73:37-43.

17. Manouguian S. Patch enlargement of the aortic valve ring by extending the aortic incision into the anterior mitral leaflet. $J$ Thorac Cardiovasc Surg. 1979;78:402-12. 